\title{
Laser Interference Lithography for Fabricating Nanowires and Nanoribbons
}

\author{
Joong-Mok Park ${ }^{1}$, Wai Leung ${ }^{1}$, Kristen Constant ${ }^{1}$, Sumit Chaudhary ${ }^{1}$, \\ Tae-Geun $\mathrm{Kim}^{2}$ and Kai-Ming $\mathrm{Ho}^{1}$ \\ ${ }^{1}$ Ames laboratory and Iowa State University, Ames, Iowa, \\ ${ }^{2}$ Korea Univerisy, Seoul, \\ ${ }^{1}$ USA \\ ${ }^{2}$ Korea
}

\section{Introduction}

Nanowires have been extensively studied for the last decade due to their superior electrical, optical, and mechanical properties when compared they are bulk. Their interesting properties are due to their simple nature (Wu B. et al., 2005). Various fabrication methods of nanowires have been in development including nanoimprint (Guo L., 2007; Chen L. et al., 2007), side electroplating (Shankar \& Raychaudhuri, 2005; Xiang et al., 2008), self assembly (Pauzauskie \& Yang, 2006), and stencil lithography (Vazquez-Mena et al., 2008) etc.

We have developed a simple, cost efficient and mass producible fabrication method for metal nanowires. First, a polymer mask is fabricated with a photosensitive material, a photoresist, using laser interference holography. Then metal is coated by physical vapor deposition (Xue et al., 2008; Kang and Guo, 2007; Kang et al., 2008), either in single or multistage depositions. After chemically removing the photoresist, parts of the metal nanowires remained on the substrate as ordered structures and parts are detached from the substrate which can be recovered for further analysis. This technique yields high quality nanowires (which are few $\mathrm{cm}$ long) either in ordered or free standing form. In this chapter, nanowires are defined as being straight as-deposited whereas nanoribbons are freestanding and are curved compared to their original configuration. The dimensions of the nanowires can be controlled by tailoring dimensions of the polymer patterns and deposition conditions such as angle and thickness.

Their structural, electrical, and optical properties are characterized by scanning electron microscopy (SEM), transmission electron microscopy (TEM), atomic force microscopy (AFM), and four point probe resistivity measurements. This fabrication method can be used for most metals (even semiconductor or insulators) to form nanowires and nanoribbons.

\section{Experiment details of fabricating nanowires}

The fabrication of nanowires is a two-step process. The first step is making polymer templates using two- or multiple-beam interference. Polymer templates can have a 1dimensional grating or 2-dimensional square structures. The templates are made on a 
transparent substrate (glass, sapphire, ITO-coated glass) or silicon wafer depending on the application. ITO-coated glass is used for electroplating metal nanowires. Glass is used when high optical transmission is required. The second step is depositing metallic thin films on top of the polymer patterns and on the substrate between the polymer channels. Because the polymer structures are well defined, large areas with rectangular cross section, long metallic nanowires are made easily. The resulting wires are $\mathrm{cm}$ long, few hundred nanometer wide and less than 100 nanometer thick.

\subsection{Interference holography}

Interference between two coherent beams is well known in classical optics (Hecht, 1987). The typical configuration of interference holography is splitting a coherent beam into two then overlapping the beams to make interference pattern as Fig. (1)a (Guo H. et al., 2007). The drawback of this setup is that the two beam intensities are close to each other and the incidence angles of the two beams are adjusted separately. When incidence angle needs to be changed, both mirrors have to be adjusted.

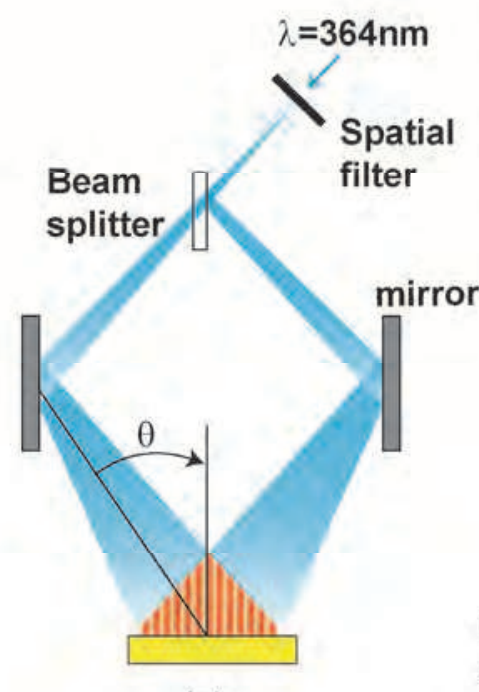

(a)

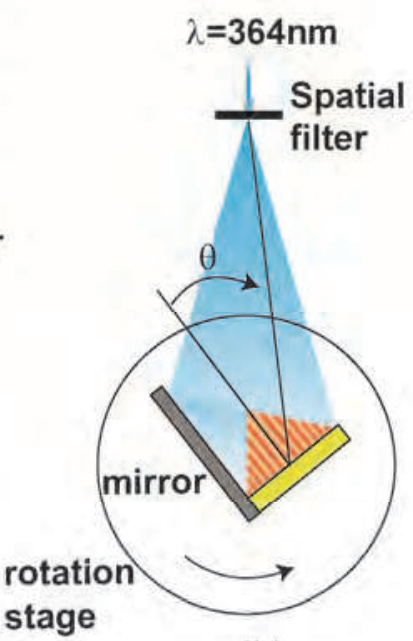

(b)

Fig. 1. Schematic diagrams of (a) two-beam interference holography with a beam splitter and (b) with Lloyd's mirror configuration.

A Lloyd's mirror setup can avoid this by using single mirror mounted perpendicular to the sample as Fig. (1)b (Onoa et al., 2005). Half of the beam is reflected from the mirror and the other half is the original beam. Because the reflecting mirror is attached to the sample, the incidence angles can be change together by rotating the sample-mirror stage. Also, mechanical rigidity reduces vibrations between interfering beams, resulting well-defined patterns even for long exposure times.

The pitch (or period, center to center distance), $\Lambda$, of the interference intensity is a function of wavelength $(\lambda)$ and incidence angle $(\theta)$ as Eq. (1). 


$$
\Lambda=\frac{\lambda}{2 \sin \theta}
$$

Ideally, the pitch can be the half of the wavelength of the laser when the incidence angle is 90 degrees. The smallest pitch is typically larger than that because the intensity decreases at high incidence angles. Also mechanical vibration between two beams, air turbulence, laser stability, dust particles and imperfect optical components limits the pitch. There is no upper limit in pitch when incidence angle approaches to zero. The sample size is determined by the interfering area. In case of a small incidence angle, the overall interference area is reduced in horizontal direction (perpendicular to the grating direction). This is because the beam size coming from the mirror is proportional to $\sin (\theta)$. Another factor determining sample size is the coherence length of the laser. A typical gas laser (Ar-ion or He-Cd) has a fairly large coherence length $(>30 \mathrm{~cm})$. So the typical exposed size (less than $10 \mathrm{~cm})$ is not limited by that.

A Lloyd's mirror configuration is as followings. An Ar-ion laser with UV prism (Coherent Inc.) is used to generate coherent light of $364 \mathrm{~nm}$ wavelength. A spatial filter, 10x ultraviolet objective lens and $10 \mu \mathrm{m}$ diameter pinhole, expands the beam and makes it homogeneous. The distance between the spatial filter and sample is $2 \mathrm{~m}$, which is far enough to ignore beam divergence and results in a large exposed area. The pitch can be adjusted by the incidence angle by rotating the stage.

The polymer templates with photo sensitive material (photoresist) are made by following process. Substrates (typically glass, Si wafer, or ITO coated glass) are prebaked $150{ }^{\circ} \mathrm{C}$ for 10 min to remove any moisture remaining after standard cleaning process. Then an adhesion promoter MCC primer (Microchem Inc.) is spin coating and baked at $120^{\circ} \mathrm{C}$ for 2 minutes on hot plate. An adhesion promoter is used to enhance a bonding between photoresist and substrate. AZ HiR 1075 photoresist (AZ-EM electronics) is spin coated on top of prebaked promoter at $4000 \mathrm{rpm}$ for $60 \mathrm{sec}$. The thickness of photoresist is controlled by adding thinner to the solution or by increasing the spin speed. The spin-coated photoresist is prebaked at 60 ${ }^{\circ} \mathrm{C}$ for $30 \mathrm{~min}$ in oven to remove solvent in the photoresist. Then the sample is mounted on a rotating sample stage and exposed. A typical dose is about $200 \mathrm{~mJ}$ (total exposure time is 30 sec). The exposed sample is baked $110^{\circ} \mathrm{C}$ for $60 \mathrm{sec}$ on hot plate and then developed in MIF 300 developer (AZ-EM electronics) for $60 \mathrm{sec}$. The developed sample is rinsed with deionized water and dried with blowing nitrogen. The thickness of photoresist including the adhesion promoter is $750 \mathrm{~nm}$ measured from a cross sectional image taken with JEOL 840 scanning electron microscope.

The intensity of the interference of two coherent beams at the sample surface is the time average of the magnitude of two interfering electric fields intensity squared as Eq. (2).

$$
I=\left\langle\left(E_{1}+E_{2}\right)^{2}\right\rangle=I_{0}[1+\cos (2 k x \sin \theta)]=I_{0}\left[1+\cos \left(\frac{2 \pi x}{\Lambda}\right)\right]
$$

The normalized intensity is illustrated in Fig. (2)a with the response line of the photoresist. The area exposed at intensity above the response line is completely removed and the area below this line remains when using a positive type photoresist. The patterns after developing are long straight lines with rectangular cross section as in Fig. (2)b. The width of individual photoresist is about half of the pitch for optical dose. It can be controlled by the exposure time and laser intensity (i.e. dose). The dose can increase or decrease the 
amplitude of the intensity curve in Fig (2)a whereas the pitch can be controlled by incidence angle and wavelength of the laser. Controlling width of the photoresist by dose is not practical because the nonlinear response of the photoresist at high or low dose.
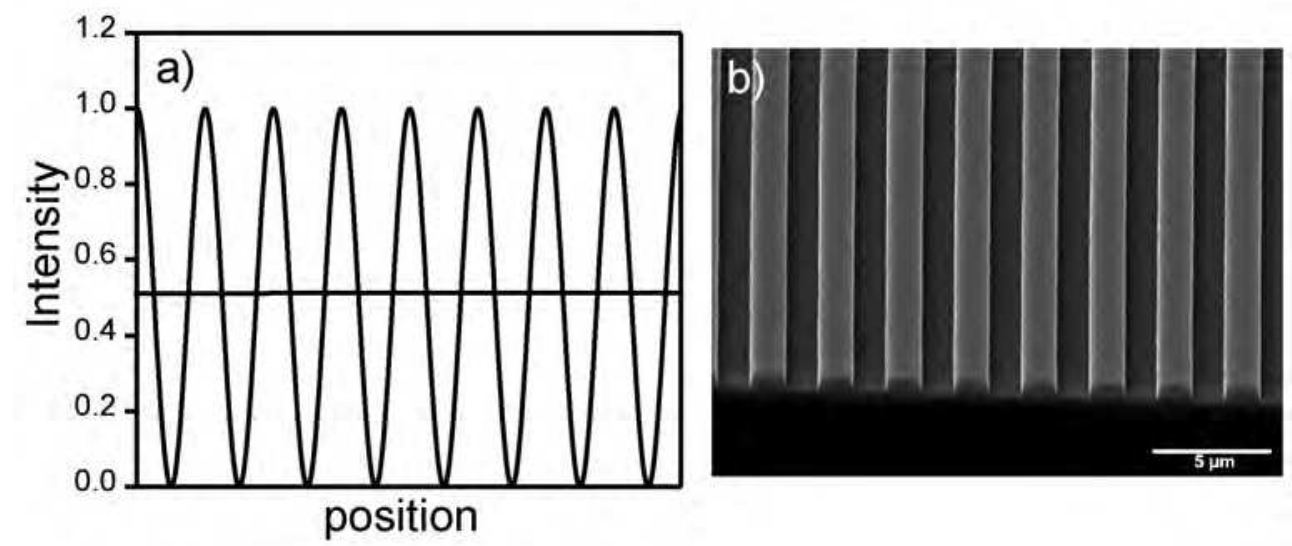

Fig. 2. (a) Normalized intensity of two beam interference and (b) SEM image of the photoresist grating made with two beam interference holography.

\subsection{Shadow deposition of metals}

After the polymer template is made with a photoresist, metal is deposited on the template with e-beam evaporation to form long nanowires. The distance between the sample and evaporation source is about $1 \mathrm{~m}$, so the deposition is approximately collimated. A quartz crystal monitor is used to monitor the deposition rate and thickness during the deposition. The deposition rate is maintained at about $1 \AA$ / sec. The pressure is below $10^{-6}$ Torr during the deposition. When deposited at normal angle, a thin metal layer is coated on top of the photoresist and also on the substrate in the channels between the photoresist bars as shown in Fig. (3)a and Fig. (4)a. If desired, deposition on the substrate can be avoided by oblique angle deposition as Fig. (3)b (Bai et al., 2007; Chen L., 2007; Chen Y. and Glidman, 2008).
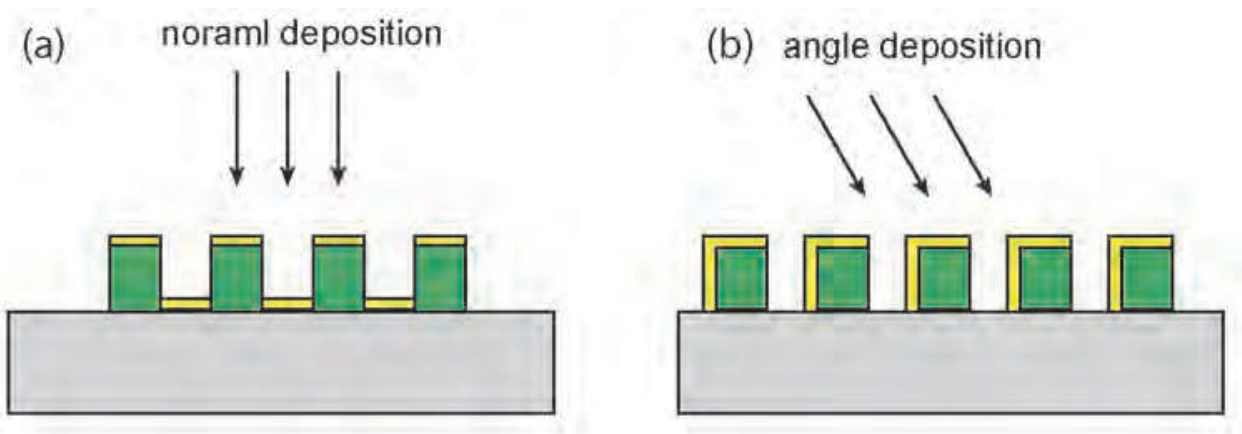

Fig. 3. Schematic diagram of two e-beam deposition methods (a) normal angle and (b) oblique angle. 
In this case, only the sidewalls and top of the photoresist are deposited by an oblique deposition angle. Partial deposition on the substrate is also possible. The deposition width on the substrate depends on the thickness and width of the photoresist and angle of deposition. The pitch, width, and thickness of the deposited materials can be controlled by the dimensions of the polymer template and metal deposition rate and time.
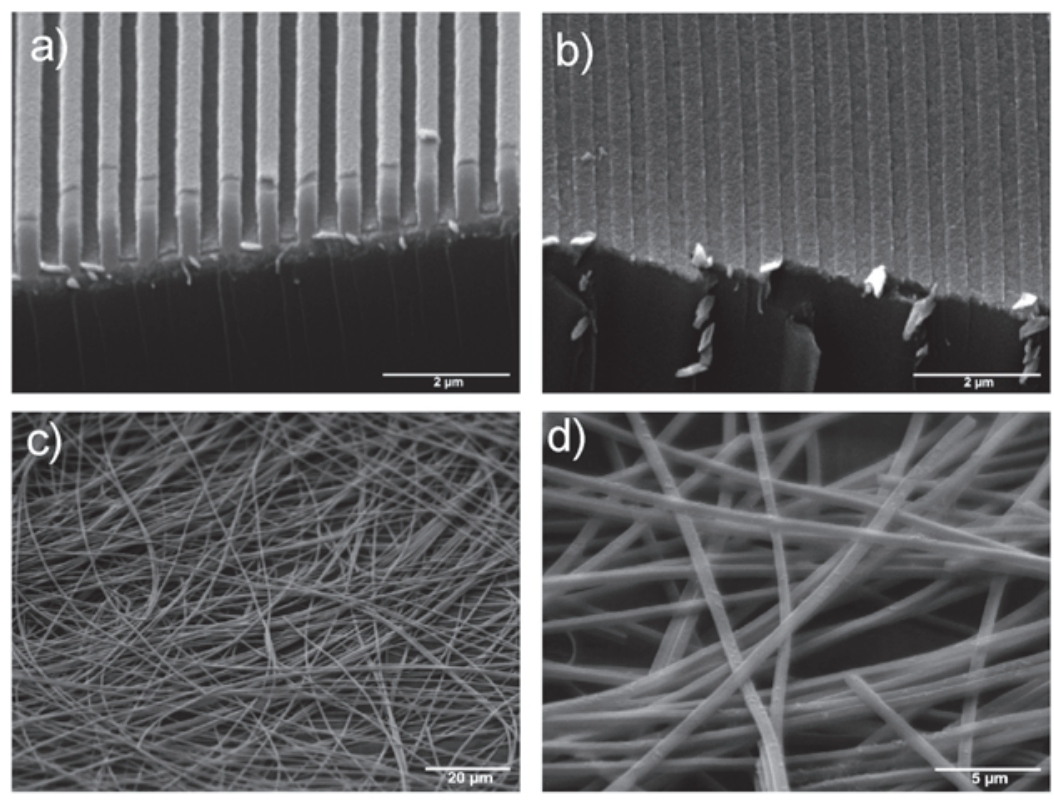

Fig. 4. SEM images of nanowires: (a) Al nanowires on the ITO coated glass substrate at normal angle deposition, (b) Al nanowires after photoresist removal. (c, d) Collected Ti nanoribbons from the IPA solution.

After deposition, the photoresist is removed by immersion in a photoresist stripper, Remover PG (Microchem Inc.), at room temperature for $30 \mathrm{~min}$ and the samples are dried with $\mathrm{N}_{2}$ gas after rinsing with IPA (isopropyl alcohol). The metal nanowires deposited on the substrate between the channels of the photoresist remain even after photoresist removal as in Fig. (4)b where as thin metallic films deposited on top of the pattern are detached during rinsing process. The detached materials can be recovered as nanoribbons which have the same thickness and roughly same width of patterns on the substrate.

Various metals $\mathrm{Ti}, \mathrm{Au}, \mathrm{Ag}, \mathrm{Cu}, \mathrm{Al}, \mathrm{Pb}$ are used as deposited materials. $\mathrm{Pb}$ has granular structures rather than uniform film. Other metals have uniform films on the photoresist or substrate.

\subsection{Phase mask and electroplating}

To fabricate large open area metal mesh structures with a single electro-deposition, a pattern is made from the interference of three diffracted beams after passing through the phase mask as in Fig. (5). The interference of three coherent beams is the superposition of three plane waves as described in Eq. (3) (De and Sevigny, 1967; Farhoud et al., 1999): 


$$
I=I_{0}[3+4 \cos (k x \sin \theta+\Delta \phi) \cos (k z(1-\cos \theta))+2 \cos (2 k x \sin \theta)]
$$

where $\mathrm{I}_{0}$ is the incident laser beam intensity, $\Delta \phi=\phi_{1}-\phi_{0}$ is the phase difference between the 1 st and the 0 th order beam, $\theta$ is the first-order diffraction angle from the normal.

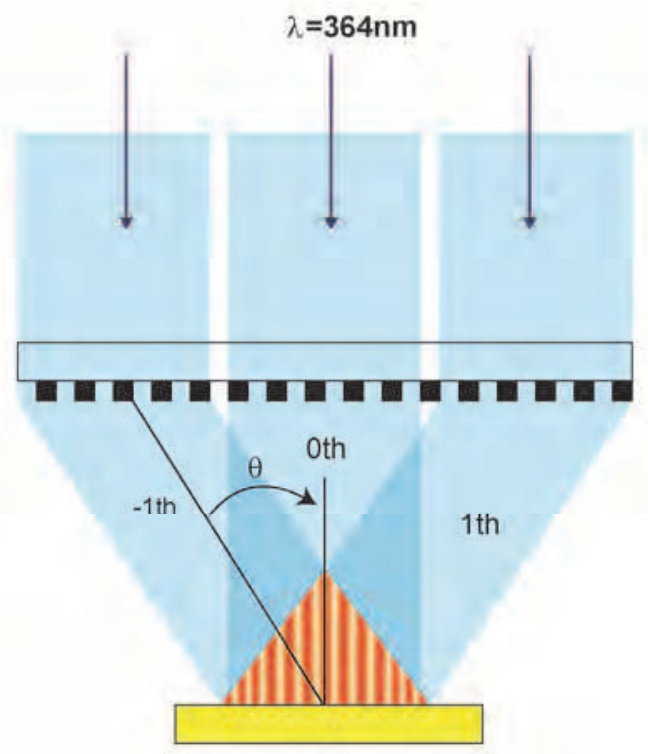

Fig. 5. Three beam interference with a diffractive phase mask.

To make metallic structures having circular or square holes, the photoresist pattern was made on indium tin oxide (ITO) coated glass to facilitate the electro-deposition of metals. $\mathrm{Cu}$ is electroplated by applying $50 \mathrm{~Hz} \mathrm{AC}$ of $10 \mathrm{~mA}$ current. Forward biases of $+5 \mathrm{~V}$ for $10 \mathrm{msec}$ and reverse $-3 \mathrm{~V}$ for $5 \mathrm{msec}$ are applied in one period. AC pulse reverse plating helps initial nucleation of $\mathrm{Cu}$ and enhances uniform film growth. The electro-deposition is stopped before overfilling the channels. Then the photoresist is removed by immersion in stripper, Remover PG, for 30 minutes.

For 2 dimensional structures, the photoresist is exposed twice with a 90 degree rotation between exposures. The double exposed photoresist of two-beam interference is a 2 dimensional array of cylindrical pillars (Fernandez et al., 1997). Figure (6)a shows the 2D intensity map for double exposure with 90 degree rotations with phase mask. The intensity across line $\mathrm{B}$ and $\mathrm{C}$ are shown in Fig (6)b. Electroplated $\mathrm{Cu}$ is the inverse structure of the photoresist when electroplating is stopped before overfilling the photoresist. The final $\mathrm{Cu}$ has circular holes after polymer removal as shown in Fig. (6)c without a phase mask, whereas a metallic mesh structure having large open square holes is made with a diffractive phase mask as in Fig. (6)d. The phase mask is made with photoresist on glass using 2-beam interference holography. The pitch of the phase mask grating was chosen as $700 \mathrm{~nm}$ to have only zero- and first-order diffractions. The $\mathrm{Cu}$ structures made with the phase mask are narrower than those made with conventional two beam holography and are close to square in cross section as shown in Fig. (6)d. 

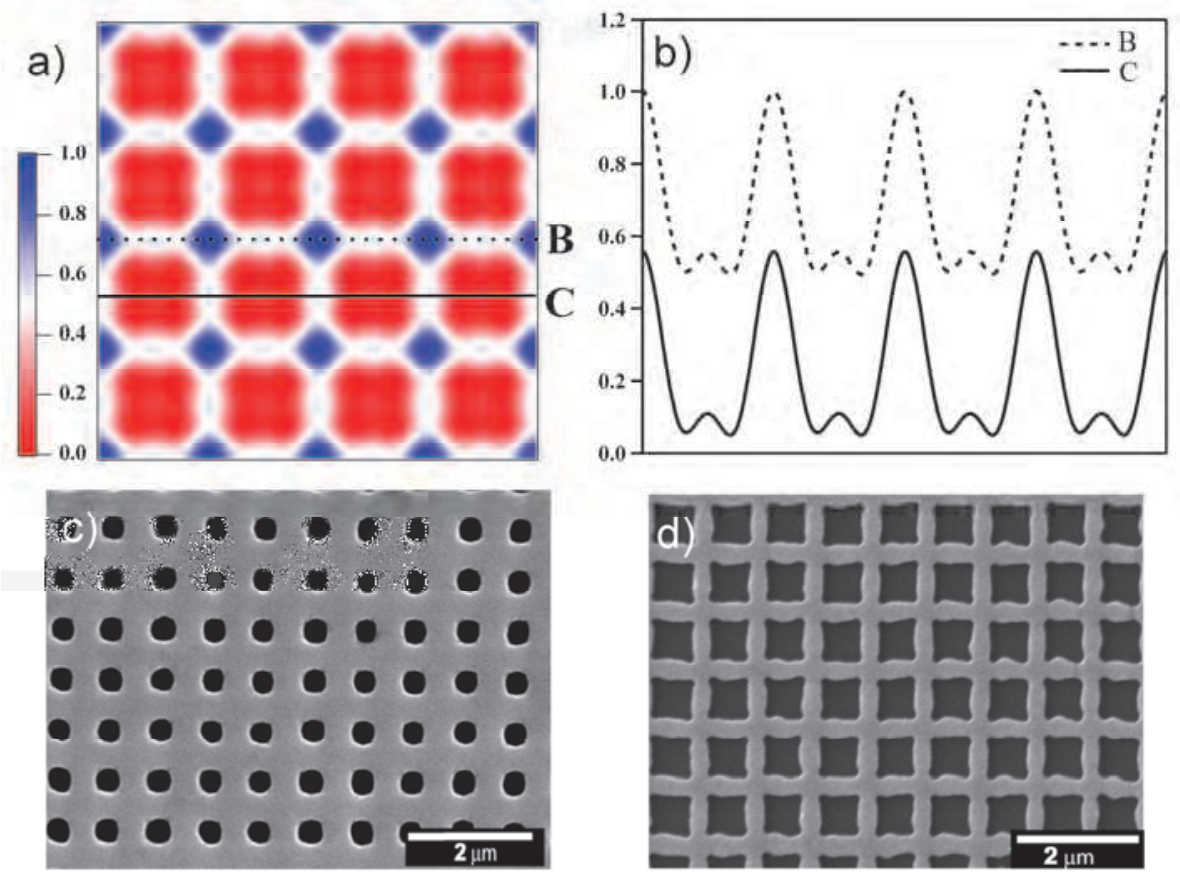

Fig. 6. (a) Intensity map of three beam interference on the substrate with double exposed with 90 degree rotation. (b) Intensity along the line B, C. (c) SEM image of Cu with two beam interference $(\mathrm{d})$ with three beam interference after photoresist removal.
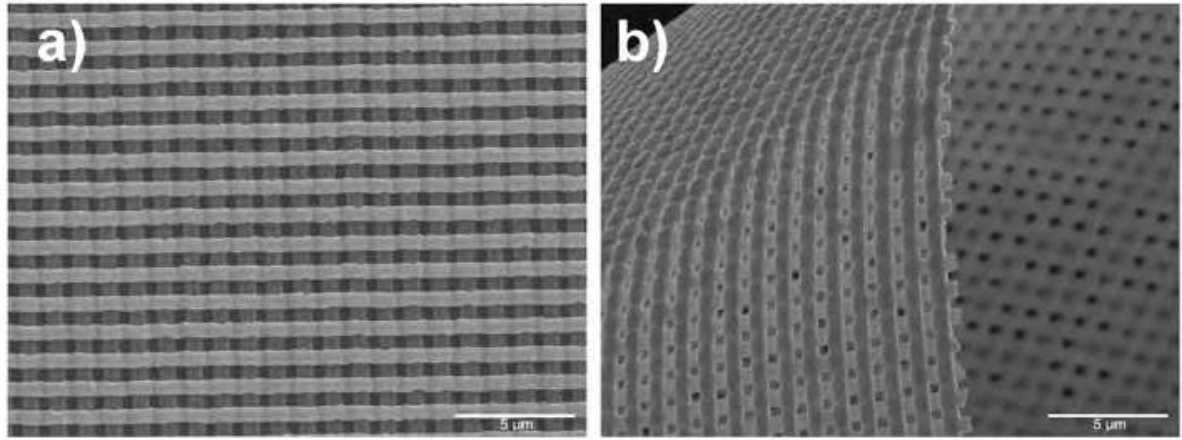

Fig. 7. (a) Cu structure electroplated between photoresist patterning. (b) Free standing $\mathrm{Cu}$ mesh peeled from the substrate.

An alternative method of making square metal structures is by two separate electrodepositions between exposures. The first $\mathrm{Cu}$ grating fills the channels of a photoresist grating made by two-beam interference. Then the second layer of photoresist is patterned 
perpendicular to the first $\mathrm{Cu}$ grating and the second $\mathrm{Cu}$ grating fills the channels of the photoresist perpendicular to the first layer. After removing photoresist, the remaining $\mathrm{Cu}$ mesh has square holes as in Fig. (7)a and can be easily detached from ITO glass making freestanding metallic mesh structures as in Fig. (7)b. Because each layer has 50\% open area, meshes made by two electro-depositions have about $25 \%$ open area.

\section{Results}

The micro structural properties, especially grain size and surface morphology, of nanowires were characterized with TEM, AFM, and SEM. Electrical conductivity was measured with a 4 point probe. Optical transmission of nanowire grating was also measured.

\subsection{Physical properties of nanowires}

To examine the grain structure of nanoribbons, TEM images were taken. Nanoribbons suspended in isopropyl alcohol were ultrasonicated for 1 min to break them into small pieces. The alcohol and broken nanoribbons were dropped onto carbon grids and vacuum assist dried. The average grain sizes of $\mathrm{Ti}$ and $\mathrm{Al}$ nanoribbons were about $10-20 \mathrm{~nm}$ by TEM as in Fig. (8)a,b. Au appears to have a larger grain size, about 50nm as Fig. (8)c. The grain

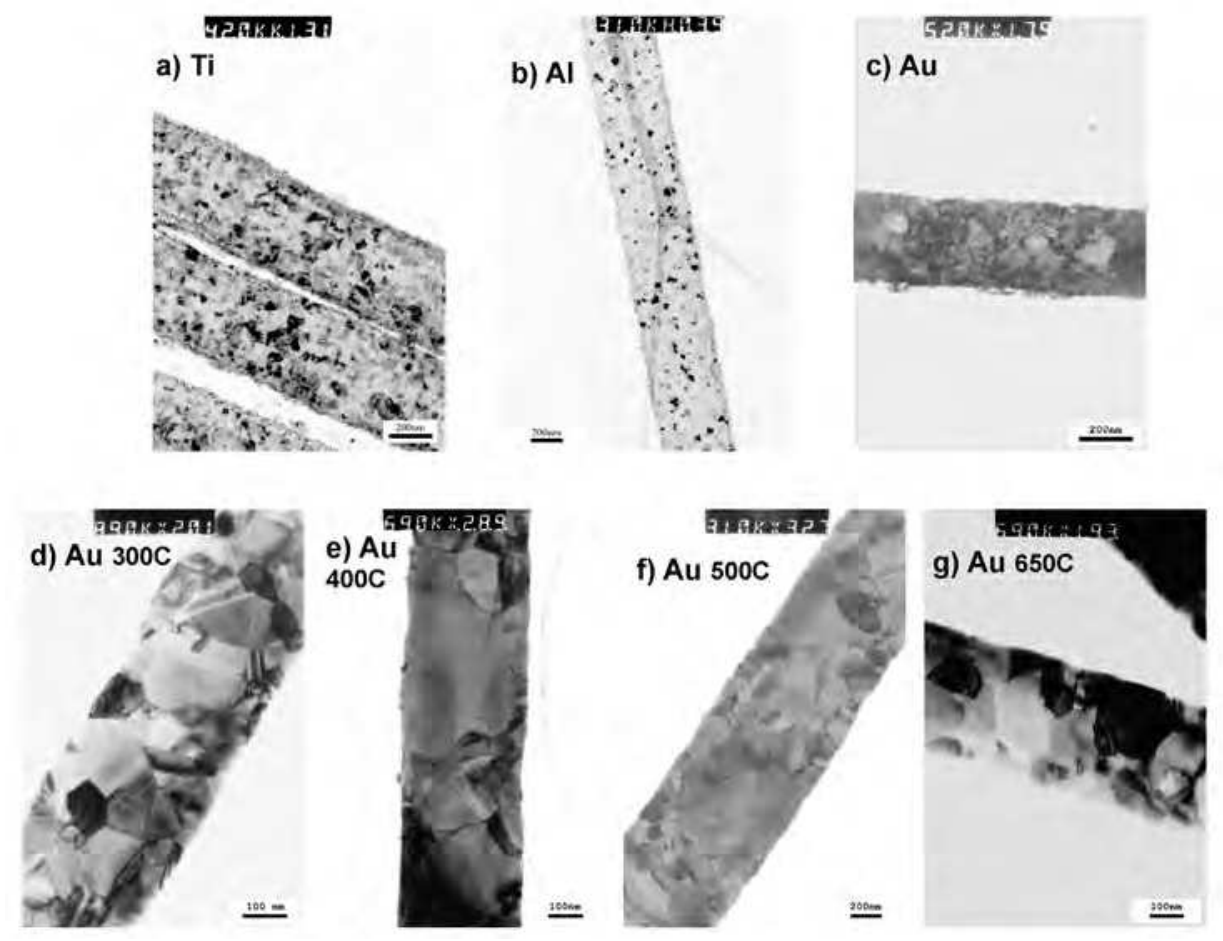

Fig. 8. Bright field TEM image of (a) Ti ribbon of $500 \mathrm{~nm} \times 100 \mathrm{~nm}$ (b) Al ribbon of $500 \mathrm{~nm} \times$ $100 \mathrm{~nm}$ (c) Au 400nm x $80 \mathrm{~nm}$ as grown. Au ribbons annealed at different temperatures (d) $300{ }^{\circ} \mathrm{C}$ for 100 hours (e) $400{ }^{\circ} \mathrm{C}$ for 48 hours (f) $500{ }^{\circ} \mathrm{C}$ for 48 hours (d) and in situ annealed at $650^{\circ} \mathrm{C}$ for 2 hours. 
size depends on various factors in the deposition process (materials, impurities, temperature, substrate etc). Thermal annealing can increase the grain size and will increase the electrical conductivity of the nanoribbons but there are some limitations to grain growth related to the small dimensions of the nanoribbons. In case of $\mathrm{Ti}$ and $\mathrm{Al}$, there was not substantial grain growth even after annealing at $600{ }^{\circ} \mathrm{C}$ for 2 hours (the melting temperatures is $660^{\circ} \mathrm{C}$ for $\mathrm{Al}$ and $1668{ }^{\circ} \mathrm{C}$ for Ti). Grain growth in $\mathrm{Au}$ nanoribbons was more substantial when annealed at temperatures well below the melting temperature $\left(1063{ }^{\circ} \mathrm{C}\right)$ as Fig. (8)d-g. Minimal grain growth of $\mathrm{Al}$ and $\mathrm{Ti}$ are due to the native oxides on the surface.

Average grain size of thin film after thermal annealing is about the same as thickness of nanowires (Thompson, 2000). Once large grains formed and further grain growing did not appear even after 48 hours annealing. The average grain size of annealed Au nanoribbons is approximately same as the thickness of nanoribbons.

AFM was used to study the surface topology of nanowires. To determine the dimensions and surface roughness, AFM images were taken of $\mathrm{Au}$ and $\mathrm{Al}$ nanowires made on $\mathrm{Si}$ substrate. AFM images show the rectangular shapes of $500 \mathrm{~nm}$ wide and $100 \mathrm{~nm}$ thick Al wires as in Fig. (9)a and $500 \mathrm{~nm}$ wide and $80 \mathrm{~nm}$ thick Au wires as in Fig. (9)c. The average surface roughness of the metal wires are about $2.5 \mathrm{~nm}$ for $\mathrm{Al}$ wires as in Fig. (9)b and $1.0 \mathrm{~nm}$ for Au wires as in Fig. (9)d.
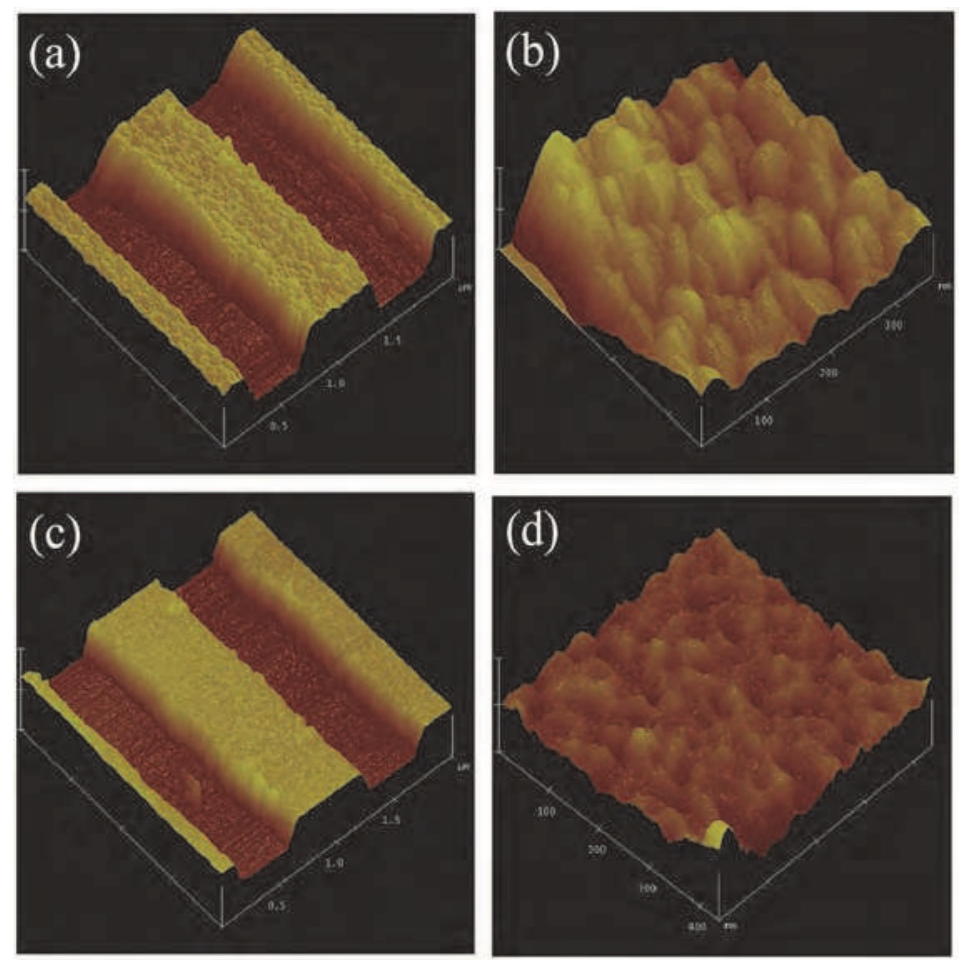

Fig. 9. (a) AFM image of $\mathrm{Al}$ wires of $500 \mathrm{~nm}$ wide and $100 \mathrm{~nm}$ thick on the Si substrate after removal of photoresist and (b) surface image. (c) Au wires of $500 \mathrm{~nm}$ width and $80 \mathrm{~nm}$ thick on the Si substrate (d) and surface image. 
Nanowires on a Si substrate have a rectangular cross section. But after annealing their upper corners were rounded. Unlike suspended by carbon grid, metal atoms migrate into Si wafer during annealing causing the reflow and turn rectangular shape into dull round corners at the edges of nanowires.

\subsection{Electric conductivity of nanowires}

Electric resistivity of metal nanowires has been measured by various authors ( $\mathrm{Wu} \mathrm{Y}$. et al., 2004; Walton et al, 2007; Peng et al., 2008; Sun et al., 2009). In this study, electric resistivity of $\mathrm{Au}$ and $\mathrm{Al}$ nanoribbons were measured with 4 point probe methods (4PP). The probes made of $4 \mathrm{Al}$ fingers on oxidized silicon wafers using a photolithography with $\mathrm{Cr}$ mask. The distance between the two inner probes was $50 \mu \mathrm{m}$. The nanoribbons in isopropyl alcohol were ultrasonicated for $1 \mathrm{~min}$ to get a homogenous dispersion. Transferring the nanoribbons on these patterns could not be accomplished by drop casting from a dispersion in isopropyl alcohol because the nanoribbons have tendency to agglomerate and do not establish an electrical path between probes. A nanoribbon network between probes is created by filtering the nanoribbons dispersed in isopropyl alcohol through a cellulose filter of $0.22 \mu \mathrm{m}$ pore size (Millipore). A continuous network of nanoribbons was obtained by vacuum filtration through the filter. The nanoribbons on the filter are stamped to the $4 \mathrm{PP}$ pattern under a pressure of $6.9 \mathrm{kPa}$ over 12 hours then the filter is dissolved with acetone. Au ribbons has a tendency for folding more easily than Al ribbons. It is not successful to get fairly large straight $\mathrm{Au}$ ribbons enough to measure resistivity. So Al ribbons are used for resistivity measurement.
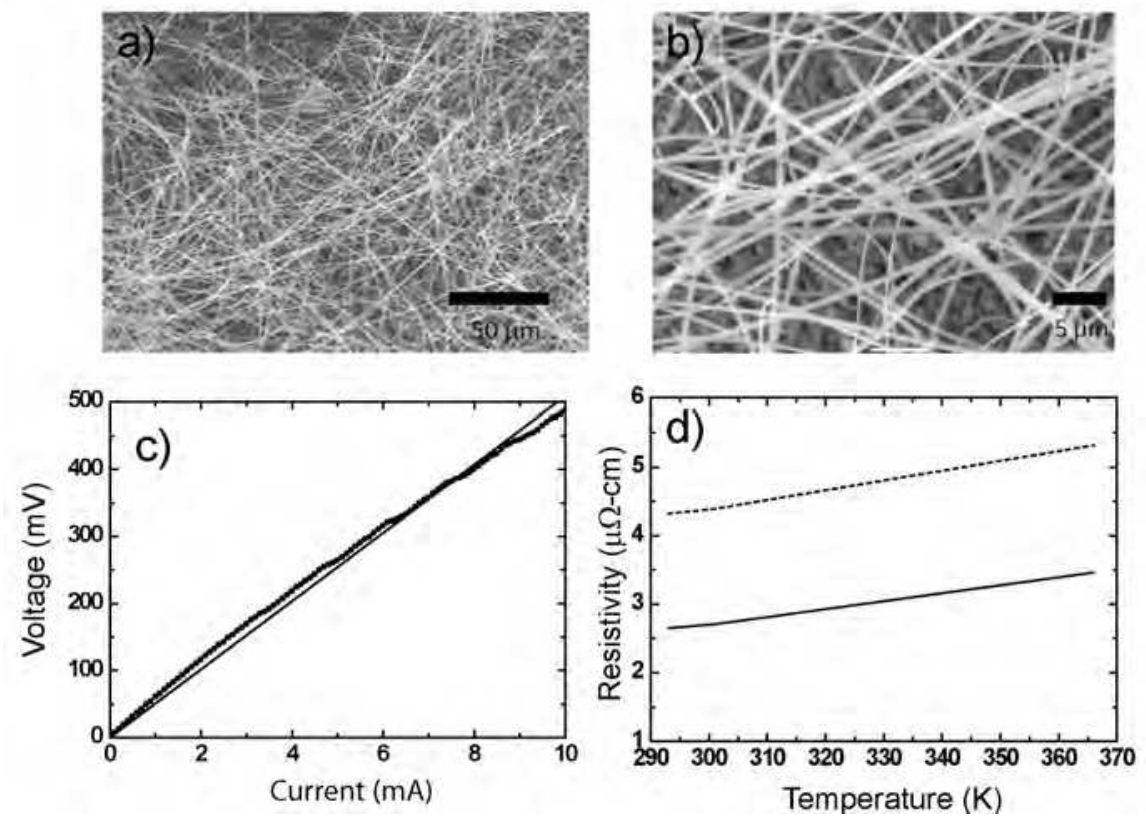

Fig. 10. $(a, b)$ SEM image of Al ribbons transferred on cellulous filter. (c) I-V curve of a single $\mathrm{Al}$ ribbon (dot) with fitted line (d) temperature dependency of resistivity of a single $\mathrm{Al}$ nanoribbon (dashed) and Al bulk (line) from $293 \mathrm{~K}$ to $366 \mathrm{~K}$. 
Figure (10)a,b shows the network of $\mathrm{Al}$ nanoribbon (300 nm width and $150 \mathrm{~nm}$ thickness) on a cellulose filter obtained after vacuum filtration. The figure illustrates that the network is dense enough to be suitable for making transparent electrodes from nanoribbons for organic light emitting devices (LEDs) and photovoltaic applications.

The I-V curve as shown in Fig. (10)c exhibits ohmic electrical behavior of the Al nanoribbon, with resistance $\sim 50 \Omega$. The resistivity of an individual nanoribbon, $\rho$ was calculated from: $\rho=A R / L$ where A is the cross sectional area of the nanoribbon and L is the electrically isolated length. Taking the width $\sim 300 \mathrm{~nm}$, thickness $\sim 150 \mathrm{~nm}$ and length $\sim 50 \mu \mathrm{m}$, the resistivity was calculated to be $\sim 4.32 \pm 0.12 \mu \Omega$-cm, which is higher than the bulk resistivity value $\sim 2.65 \mu \Omega$-cm (Lide, 1997). Figure (10)d shows the temperature dependence of resistance of a single nanoribbon. The resistance is found to increase with temperature for both $\mathrm{Al}$ bulk and nanoribbons, however slope $(\mathrm{dR} / \mathrm{dT})$ is slightly different for the two cases. The resistivity of nanowires increases when the electron mean free path is larger than dimension of nanowires due to surface scattering and grain boundary scattering of electrons (Mani et al., 2006). Additionally, $\mathrm{Al}$ has a thin $(\sim 10 \mathrm{~nm})$ native oxide layer on the surface. Thin insulating aluminum oxide layer can not be ignored in thin films and nanowires. The temperature dependence of resistivity can be explained by electron-phonon scattering (Bid et al., 2006). The $\mathrm{dR} / \mathrm{dT}$ of nanowires is smaller than that of bulk because of the Debye temperature change.

\subsection{Optical transmission of nanowires grating}

Specular transmission data was collected for $\mathrm{Al}$ nanowire grating made on a glass substrate with fiber coupled spectrometer (PCI S2000, Ocean optics) for transmission measurement in the visible to near IR spectrum range $(400 \mathrm{~nm}$ to $1000 \mathrm{~nm})$ as Fig. (11). Al nanowires have period of $700 \mathrm{~nm}$ and thickness of $150 \mathrm{~nm}$ made on $200 \mu \mathrm{m}$ thick glass substrate for transmission measurements. Narrow $100 \mathrm{~nm}$ width of $\mathrm{Al}$ nanowires are made with three angle deposition with shadow mask lithography (Park et al., 2010). Wavelengths greater than $\sim 700 \mathrm{~nm}$, which is the period of the grating, have higher transmission also there is a minima around $530 \mathrm{~nm}$. This is the second order diffraction of the glass-nanowire interface. Multiplying the pitch by the refractive index of glass, 1.52, and dividing by 2 yields $532 \mathrm{~nm}$.
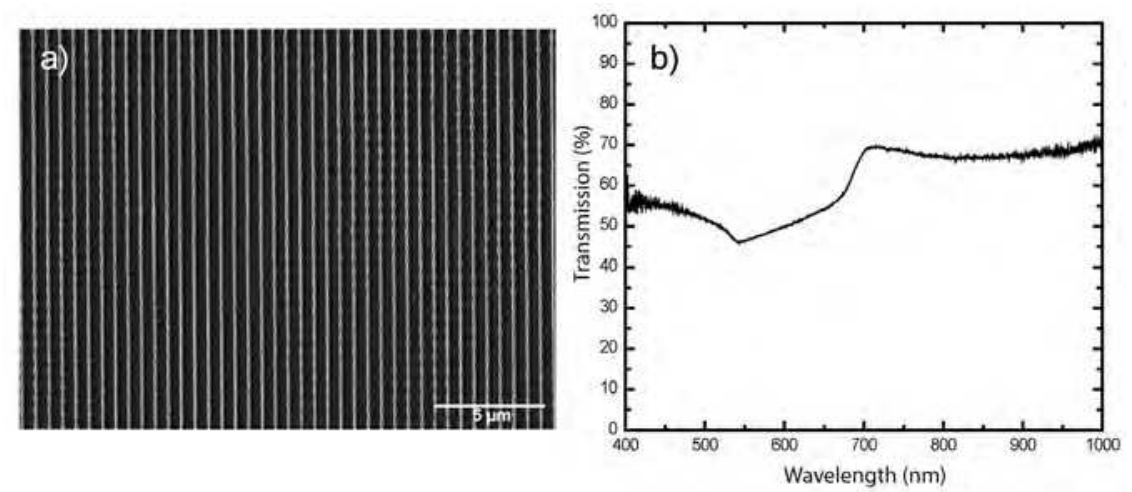

Fig. 11. (a) SEM image of Al grating of $700 \mathrm{~nm}$ pitch. Individual Al nanowires have $150 \mathrm{~nm}$ height and $100 \mathrm{~nm}$ width. (b) Transmission spectra of Al nanowire grating made on glass substrate. 
Metallic nanowires having high transmission in visible spectrum range with high electric conductivity can replace indium tin oxide (ITO) as transparent conducting electrodes.

\section{Conclusion}

In summary, we fabricated metallic nanowires and nanoribbons by e-beam assisted metal depositions and electroplating with photoresist patterns made by interference holography of multiple beams. This fabrication method can produce well-defined, rectangular crosssectioned nanowires with dimensions of a few $\mathrm{cm}$ long and submicron width on substrates as well as free-standing forms. Nanowires made on substrates can be further processed to make desired cross-mesh structures. Suspended nanoribbons in the IPA can be further processed or stamped as random meshes. Structural properties of metallic nanowires and nanoribbons are characterized using SEM, AFM, and TEM. Electrical resistivity of $\mathrm{Al}$ nanoribbons is also measured with $4 \mathrm{PP}$. Potential applications of one-dimensional nanostructures are electrodes for cross-bar electronic devices like liquid crystals, and transparent electrodes for light-emitting-diodes and photovoltaic cells.

$\mathrm{Cu}$ metallic mesh structures are fabricated by electrochemical deposition on photoresist templates. The metallic mesh made with diffractive phase mask has large open area than that made with two beam interference. Also, the metal mesh is flexible and peeled off from the substrate to make free standing. This method is applicable to the mass production of high quality metallic nanostructures and applications requiring large areas without the need for clean room or e-beam systems.

\section{Acknowledgment}

This work is supported by the Division of Materials Sciences and Engineering, Basic Energy Sciences, US Department of Energy. The Ames Laboratory is operated by Iowa State University for the Office of Science, U.S. Department of Energy under Contract DE-AC0207CH11358. This work also was supported by a Korea Research Foundation grant funded by the Korean Government (MOEHRD) (KRF-2008-D00074).

\section{References}

Bai, John G; Chang, Cheng-Ling; Chung, Je-Hyung and Lee, Kyong-Hoon (2007). Shadow edge lithography for nanoscale patterning and manufacturing, Nanotechnology, Vol. 18, (2007), pp. 405307 (8pp).

Bid, Aveek; Bora, Achyut and Raychaudhuri, A. K. (2006). Temperature dependence of resistivity of metallic nanowires of diameter $>15 \mathrm{~nm}$ : Applicability of BlochGruneisen theorem, Phys. Rev. B, Vol. 74, (2006), pp. 035426 (8pp).

Chen, Lei; Wang, Jian; Frank, Jim; Deng, Walters, Xuegong; Buonanno, Mike; Tai, Stephen and Liu, Xiaoming (2007). Large flexible nanowire grid visible polarizer made by nanoimprint lithography, Appl. Phys. Lett., Vol. 90, (2007), pp. 063111 (3pp).

Chen, $\mathrm{Yu}$ and Goldman, A. M. (2008). A simple approach to the formation of ultranarrow metal wires, J. Appl. Phys., Vol. 103, (2008), pp. 054312 (4pp).

De, Manoranjan and Sevigny, Landre (1967). Three-Beam Holographic Interferometry, Appl. Opt., Vol. 6, (1967), pp. 1665-1671. 
Farhoud, Maya; Ferrera, Juan; Lochtefeld, Anthony J.; Murphy, T. E.; Schattenburg, Mark L.; Carter, J.; Ross, C. A. and Smith, Henry I. (1999). Fabrication of $200 \mathrm{~nm}$ period nanomagnet arrays using interference lithography and a negative resist, J. Vac. Sci. Technol. B Vol. 17, (1999), 3182-3185.

Fernandez, A.; Decker, J. Y.; Herman, S. M.; Phillion, D. W.; Sweeney, D. W. and Perry M. D. (1997). Methods for fabricating arrays of holes using interference lithography, $J$. Vac. Sci. Technol. B Vol. 15.6., (1997), pp. 2439-2443.

Guo, H. C.; Nau, D.; Radke, A.; Zhang, X. P.; Stodolka, J.; Yang, X. L.; Tijhodeev, S. G.; Gippius, N. A.; Giessen H. (2005). Large-area metallic photonic crystal fabrication with interference lithography and dry etching, App. Phys. B, Vol. 81, (2005), pp. 271 -275.

Guo, L. Jay (2007). Nanoimprint Lithography: Methods and Material Requirements, Adv. Mater., Vol. 19, (2007), pp. 495-513.

Hecht, Eugene (1987). Optics (2nd Edition), Addison-Wesley, ISBN 0-201-11609-X, Reading, Massachusetts.

Kang, Myung-Gyu and Guo, L. Jay (2007). Nanoimprinted Semitransparent Metal Electrodes and Their Application in Organic Light-Emitting Diodes, Adv. Mater., Vol. 19, (2007), pp. 1391-1396.

Kang, Myung-Gyu; Kim, Myung-Su, Kim, Jinsang and Guo, L. Jay (2008). Organic Solar Cells Using Nanoimprint Transparent Metal Electrodes, Adv. Mater., Vol. 20, (2008), pp. 1-6.

Lide, R. David (1997). CRC Handbook of Chemistry and Physics (87th Edition), Tayler and Francis, ISBN 978-0849304873, Boca Raton, FL.

Mani, Sathya; Saif, Taher andHan, Jong H. (2006). Effect of Annealing on the Conductivity of Electroless Deposited Ni Nanowires and Films, IEEE Trans. on Nanotechnology, Vol. 5, No. 2, (2006), pp. 138-141.

Onoa, G Bibiana; O'Reilly, Thomas B; Walsh, Michael E and Smith, Henry I (2005). Bulk production of singly dispersed carbon nanotubes with prescribed lengths, Nanotechnology, Vol. 16, No.12, (2005), pp. 2799-2803

Park, Joong-Mok; Nalwa, Kanwar Singh; Leung, Wai; Constant, Kristen; Chaudhary, Sumit and Ho, Kai-Ming (2010). Fabrication of metallic nanowires and nanoribbons using laser interference lithography and shadow lithography, Nanotechnology, Vol. 21, (2010), pp. 215301 (6pp).

Pauzauskie, Peter J. \& Yang, Peidong (2006). Nanowire Photonics, Materials today, Vol.9, No. 10, (2006), pp. 36-45.

Peng, Yong; Cullis, Tony and Inkson, Beverley (2008). Accurate electrical testing of individual gold nanowires by in situ scanning electron microscope nanomanipulators, Appl. Phys. Lett., Vol. 93, (2008), pp. 183112 (3pp).

Shankar, K. Shantha and Raychaudhuri, A.K. (2005). Fabrication of nanowires of multicomponent oxides: Review of recent advances, Materials Science and Engineering C, Vol. 25, (2005), pp. 738-751.

Sun, Tik; Tao, Ba; Warren, Andrew P.; Barmak, Katayun; Toney, Michael F.; Peale, Robert E. and Coffey, Kevin R. (2009). Dominant role of grain boundary scattering in the resistivity of nanometric Cu film, Phys. Rev. B, Vol. 79, (2009), pp. 041402 (4pp).

Thompson, C. V. (2000). Structure Evolution during Processing Polycrystalline Films, Annu. Rev. Mater. Sci.,Vol. 30, (2000), pp. 159-190.

Vazquez-Mena, O.; Savu, V.; Sidler, K.; Villanueva, G.; Boogaart, M. A. F. van den ; Brugger J. (2008). Sub-100 nm-scale Aluminum Nanowires by Stencil Lithography: 
Fabrication and Characterization, Proceedings of the 3rd IEEE Int. Conf. on Nano/Micro Engineered and Molecular Systems, January 6-9, 2008, Sanya, China, pp. 807-811.

Walton, A S; Allen, C S; Critchley, K; Gorzny, M L; McKendry, J E; Brydson, R M D; Hickey, B J and Evans, S D (2007). Four-probe electrical transport measurements on individual metallic nanowires, Nanotechnology, Vol. 18, No. 40, (2007), pp. 065204 (6pp).

Wu, Bin; Heidelberg, Andreas and Boland, John J. (2005). Mechanical properties of ultrahigh strength gold nanowires, Nature Materials, Vol. 4, (2005), pp. 525-529

Wu, Yue; Xiang, Jie; Yang, Chen; Lu, Wei \& Lieber, Charles M. (2004). Single-crystal metallic nanowires and metal/semiconductor nanowire heterostructures, Nature, Vol. 430, No.1, (2004), pp. 61- 65.

Xiang, Chenxiang; Kung, Sheng-Chin; Taggart, David K.; Yang, Fan; Thompson, Michael A.; Guell, Aleix G.; Yang, Yongan and Penner, Reginald M. (2008). Lithographically Patterned Nanowire Electrodeposition: A Method for Patterning Electrically Continuous Metal Nanowires on Dielectrics, ACS Nano, Vol. 2, No. 9, (August 2008), pp. 1939-1949.

Xue, Mianqi; Yang, Yanlian and Cao, Tingbing (2008). Well-Positioned Metallic Nanostructures Fabricated by Nanotransfer Edge Printing, Adv. Mater., Vol. 20, (2008), pp. 596-600. 


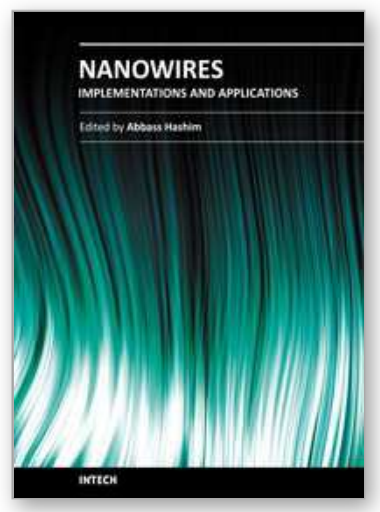

\author{
Nanowires - Implementations and Applications \\ Edited by Dr. Abbass Hashim
}

ISBN 978-953-307-318-7

Hard cover, 538 pages

Publisher InTech

Published online 18, July, 2011

Published in print edition July, 2011

This potentially unique work offers various approaches on the implementation of nanowires. As it is widely known, nanotechnology presents the control of matter at the nanoscale and nanodimensions within few nanometers, whereas this exclusive phenomenon enables us to determine novel applications. This book presents an overview of recent and current nanowire application and implementation research worldwide. We examine methods of nanowire synthesis, types of materials used, and applications associated with nanowire research. Wide surveys of global activities in nanowire research are presented, as well.

\title{
How to reference
}

In order to correctly reference this scholarly work, feel free to copy and paste the following:

Park Joong Mok, Wai Leung, Kristen Constant, Tae-Geun Kim and Kai-Ming Ho (2011). Laser Interference Lithography and Shadow Lithography for Fabricating Nanowires and Nanoribbons, Nanowires Implementations and Applications, Dr. Abbass Hashim (Ed.), ISBN: 978-953-307-318-7, InTech, Available from: http://www.intechopen.com/books/nanowires-implementations-and-applications/laser-interferencelithography-and-shadow-lithography-for-fabricating-nanowires-and-nanoribbons

\section{INTECH}

open science | open minds

\section{InTech Europe}

University Campus STeP Ri

Slavka Krautzeka 83/A

51000 Rijeka, Croatia

Phone: +385 (51) 770447

Fax: +385 (51) 686166

www.intechopen.com

\section{InTech China}

Unit 405, Office Block, Hotel Equatorial Shanghai

No.65, Yan An Road (West), Shanghai, 200040, China

中国上海市延安西路65号上海国际贵都大饭店办公楼405单元

Phone: +86-21-62489820

Fax: +86-21-62489821 
(C) 2011 The Author(s). Licensee IntechOpen. This chapter is distributed under the terms of the Creative Commons Attribution-NonCommercialShareAlike-3.0 License, which permits use, distribution and reproduction for non-commercial purposes, provided the original is properly cited and derivative works building on this content are distributed under the same license. 